\title{
RICCI COEFFICIENTS OF ROTATION OF GENERALIZED FINSLER SPACES
}

\author{
SVETISLAV M. MINČIĆ, MIĆA S. STANKOVIĆ, AND MILAN LJ. ZLATANOVIĆ
}

Received 29 October, 2014

\begin{abstract}
Generalized Finsler space $G F_{n}$ is a differentiable $N$-dimensional manifold with nonsymmetric basic tensor $g_{i j}(x, \dot{x})$ defined by condition (1.2). Using the basic tensor, by (1.4) a non-symmetric connection $P^{*}$ is defined, and also four kinds of covariant derivative in the Rund's sense and five curvature tensors are obtained (Section 1).

In Section 2 two kinds of Ricci coefficients of rotation are defined and their properties are exposed. Also, integrability conditions of the equation expressing covariant derivatives of the congruence vector by means of coefficients of rotation, are obtained.

In Section 3 a geodesic mapping of two spaces $G F_{n}$ and $G \bar{F}_{n}$ is defined and some it's properties are proved.

In Section 4 some invariants of such mappings in relation with the coefficients of rotation are studied.
\end{abstract}

2010 Mathematics Subject Classification: 53A45, 53B05, 53B40

Keywords: generalized Finsler spaces, non-symmetric connections, Ricci coefficients of rotation, geodesic mappings, projective ivariants

\section{INTRODUCTION}

The generalized Finsler space $\left(G F_{n}\right)$ is a differentiable manifold with non-symmetric basic tensor field $g_{i j}\left(x^{1}, \ldots, x^{N}, \dot{x}^{1}, \ldots, \dot{x}^{N}\right) \equiv g_{i j}(x, \dot{x})$, where

$$
g_{i j}(x, \dot{x}) \neq g_{j i}(x, \dot{x}), \quad\left(g=\operatorname{det}\left(g_{i j}\right) \neq 0, \dot{x}=d x / d t\right) .
$$

Based on (1.1), the symmetric and anti-symmetric part of $g_{i j}$ are defined

$$
g_{\underline{i j}}=\frac{1}{2}\left(g_{i j}+g_{j i}\right), \quad g_{i j}=\frac{1}{2}\left(g_{i j}-g_{j i}\right) .
$$

Following [18], in our notation, hold

$$
\text { a) } g_{\underline{i j}}(x, \dot{x})=\frac{1}{2} \frac{\partial^{2} F^{2}(x, \dot{x})}{\partial \dot{x}^{i} \partial \dot{x}^{j}}, \quad \text { b) } \frac{\partial g_{i j}}{\partial \dot{x}^{k}}=0,
$$

Research supported by Ministry of Education Science and Technological Development, Republic of Serbia, Grant No. 174012. 
where $F(x, \dot{x})$ is a metric function in $G F_{n}$, having the properties known from the theory of usual Finsler spaces $\left(F_{n}\right)$. A lot of research papers (see for example $[1-4,6,11,14-19,21,22])$ are dedicated to the theory of Finsler spaces and their generalizations. The discussed structure is a particular case of the Eisenhart-Lagrange approach which is studied in [12] (Chap. 8).

The lowering and the raising of indices are defined by the tensors $g_{i j}$ and $h^{i j}$ respectively, where $h^{i j}$ satisfy equation

$$
g_{\underline{i j}} h^{j k}=\delta_{i}^{k}, \quad\left(\underline{g}=\operatorname{det}\left(g_{\underline{i j}}\right) \neq 0\right) .
$$

Generalized Cristoffel symbols of the $1^{s t}$ and the $2^{\text {nd }}$ kind are defined:

$$
\begin{aligned}
& \gamma_{i . j k}=\frac{1}{2}\left(g_{j i, k}-g_{j k, i}+g_{i k, j}\right) \neq \gamma_{i . k j}, \\
& \gamma_{j k}^{i}=h^{i p} \gamma_{p . j k}=\frac{1}{2} h^{i p}\left(g_{j p, k}-g_{j k, p}+g_{p k, j}\right) \neq \gamma_{k j}^{i},
\end{aligned}
$$

where, e.g., $g_{j i, k}=\partial g_{j i} / \partial x^{k}$.

Then we have

$$
\gamma_{j k}^{p} g_{i \underline{p}}=\gamma_{s . j k} h^{p s} g_{\underline{i p}}=\gamma_{s . j k} \delta_{i}^{* s}=\gamma_{i . j k} .
$$

Introducing a tensor $C_{i j k}$ like as at space $F_{n}$, we have

$$
C_{i j k}(x, \dot{x}) \stackrel{\text { def }}{=} \frac{1}{2} g_{i j, \dot{x}^{k}}=\frac{1}{2} g_{i j, \dot{x}^{k}}=\frac{1}{4} F_{\dot{x}^{i} \dot{x}^{j} \dot{x}^{k}} .
$$

We see that $C_{i j k}$ is symmetric in relation to each pair of indices. Also, we have

$$
C_{j k}^{i} \stackrel{\text { def }}{=} h^{i p} C_{p j k}=h^{i p} C_{j p k}=h^{i p} C_{j k p} .
$$

With help of coefficients

$$
P_{j k}^{i}=\gamma_{j k}^{i}-C_{j p}^{i} \gamma_{s k}^{p} \dot{x}^{* s} \neq P_{k j}^{i}
$$

one obtains coefficients of a non-symmetric affine connections in the Rund's sence (see [17, 18]):

$$
\begin{gathered}
P_{j k}^{* i}=\gamma_{j k}^{i}-h^{i q}\left(C_{j q p} P_{k s}^{p}+C_{k q p} P_{j s}^{p}-C_{j k p} P_{q s}^{p}\right) \dot{x}^{* s} \neq P_{k j}^{* i}, \\
P_{i . j k}^{*}=P_{j k}^{* r} g_{\underline{i r}}=\gamma_{i . j k}-\left(C_{i j p} P_{k s}^{p}+C_{i k p} P_{j s}^{p}-C_{j k p} P_{i s}^{p}\right) \dot{x}^{* s} \neq P_{i . k j}^{*} .
\end{gathered}
$$

In $G F_{n}$ we denote anti-symmetric and symmetric part for a connection $P^{*}$ respectively:

$$
\text { a) } T_{j k}^{* i}(x, \dot{x})=\frac{1}{2}\left(P_{j k}^{* i}-P_{k j}^{* i}\right)=\frac{1}{2}\left(\gamma_{j k}^{i}-\gamma_{k j}^{i}\right), \quad \text { b) } P_{\underline{j k}}^{* i}=\frac{1}{2}\left(P_{j k}^{* i}+P_{k j}^{* i}\right),
$$

where $T_{j k}^{* i}$ is the torsion tensor of the connection $P_{j k}^{* i}$. 
We define four kinds of covariant derivative of a tensor in the space $G F_{n}$. For example, for a tensor $a_{j}^{i}(x, \xi)$ :

$$
\begin{aligned}
& a_{j \mid m}^{i}(x, \xi)=a_{j, m}^{i}+a_{j, \dot{p}}^{i} \xi_{, m}^{p}+P_{p m}^{* i} a_{j}^{p}-P_{j m}^{* p} a_{p}^{i}, \\
& a_{\substack{1 \\
i}}^{i}(x, \xi)=a_{j, m}^{i}+a_{j, \dot{p}}^{i} \xi_{, m}^{p}+P_{m p}^{* i} a_{j}^{p}-P_{m j}^{* p} a_{p}^{i}, \\
& a_{j \mid m}^{i}(x, \xi)=a_{j, m}^{i}+a_{j, \dot{p}}^{i} \xi_{, m}^{p}+P_{p m}^{* i} a_{j}^{p}-P_{m j}^{* p} a_{p}^{i}, \\
& a_{\substack{j \mid m \\
i}}^{i}(x, \xi)=a_{j, m}^{i}+a_{j, \dot{p}}^{i} \xi_{, m}^{p}+P_{m p}^{* i} a_{j}^{p}-P_{j m}^{* p} a_{p}^{i},
\end{aligned}
$$

where $\xi(x)$ is an arbitrary tangent vector in the tangent space $T_{n}(x)$, and $a_{j, \dot{p}}^{i}=$ $\partial a_{j}^{i} / \partial \dot{x}^{p}$.

In the work [10] we obtain 10 Ricci type identities in the general case for a tensor $a_{t_{1} \ldots t_{v}}^{r_{1} \ldots r_{u}}(x, \xi)$ and three curvature tensors of $G F_{n}$ :

$$
\begin{aligned}
\widetilde{K}_{j m n}^{i}= & P_{j m, n}^{* i}-P_{j n, m}^{* i}+P_{j m}^{* p} P_{p n}^{* i}-P_{j n}^{* p} P_{p m}^{* i}+P_{j m, \dot{s}}^{* p} \xi_{, n}^{* s}-P_{j n, \dot{s}}^{* p} \xi_{, m}^{* s}, \\
\widetilde{K}^{i}{ }_{j m n} & =P_{m j, n}^{* i}-P_{n j, m}^{* i}+P_{m j}^{* p} P_{n p}^{* i}-P_{n j}^{* p} P_{m p}^{* i}+P_{m j, \dot{s}}^{* p} \xi_{, n}^{* s}-P_{n j, \dot{s}}^{* p} \xi_{, m}^{* s}, \\
\widetilde{K}_{3}^{i}{ }_{j m n}= & P_{j m, n}^{* i}-P_{n j, m}^{* i}+P_{j m}^{* p} P_{n p}^{* i}-P_{n j}^{* p} P_{p m}^{* i}+P_{n m}^{* p}\left(P_{p j}^{* i}-P_{j p}^{* i}\right) \\
& +P_{j m, \dot{s}}^{* p} \xi_{, n}^{* s}-P_{n j, \dot{s}, m}^{* p} \xi_{, m}^{* s} .
\end{aligned}
$$

The magnitudes $\widetilde{K}_{t}^{i}{ }_{j m n}, t=1,2,3$ are tensors and we call them curvature tensors of the first, the second and the third kind respectively.

In the work [9] we use the third and the fourth kind of covariant derivative (1.4), and in that manner one gets 10 new Ricci type identities. In these identities appear the same quantities $\underset{t}{\widetilde{K}_{j m n}^{i}}, t=1,2,3 ; \underset{t}{\widetilde{A}_{j m n}^{i}}, t=1, \ldots, 15$, but in different distribution. Only in the last case appears a new curvature tensor (of the fourth kind) $\underset{4}{\widetilde{K}}$ :

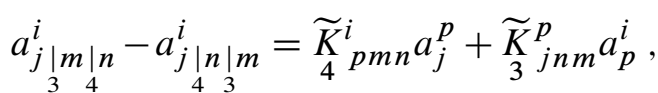

where

$$
\begin{aligned}
\widetilde{K}_{4}^{i}{ }_{j m n}= & P_{j m, n}^{* i}-P_{n j, m}^{* i}+P_{j m}^{* p} P_{n p}^{* i}-P_{n j}^{* p} P_{p m}^{* i}+P_{j m, \dot{s}}^{* p} \xi_{, n}^{* s}-P_{n j, \dot{s}, m}^{* p} \xi_{, m}^{* s} \\
& +P_{m n}^{* p}\left(P_{p j}^{* i}-P_{j p}^{* i}\right) .
\end{aligned}
$$

Denoting by semicolon (;) the covariant derivative with respect to the symmetric connection $P_{j k}^{* i}$, then using (1.3) we have

$$
\widetilde{K}_{1}^{i}{ }_{j m n}=\widetilde{K}_{j m n}^{i}+T_{j m ; n}^{* i}-T_{j n ; m}^{* i}+T_{j m}^{* p} T_{p n}^{* i}-T_{j n}^{* p} T_{p m}^{* i},
$$




$$
\begin{aligned}
& \widetilde{K}_{2 j m n}^{i}=\widetilde{K}_{j m n}^{i}+T_{m j ; n}^{* i}-T_{n j ; m}^{* i}+T_{m j}^{* p} T_{n p}^{* i}-T_{n j}^{* p} T_{m p}^{i}, \\
& \widetilde{K}_{3 j m n}^{i}=\widetilde{K}_{j m n}^{i}+T_{j m ; n}^{* i}-T_{n j ; m}^{* i}+T_{j m}^{* p} T_{n p}^{* i}-T_{n j}^{* p} T_{p m}^{i}+T_{m n}^{* p} T_{j p}^{* i}, \\
& \widetilde{K}_{4}^{i}{ }_{j m n}=\widetilde{K}_{j m n}^{i}+T_{j m ; n}^{* i}-T_{n j ; m}^{* i}+T_{j m}^{* p} T_{n p}^{* i}-T_{n j}^{* p} T_{p m}^{* i}-T_{m n}^{* p} T_{j p}^{* i},
\end{aligned}
$$

where $\widetilde{K}_{j m n}^{i}$ is the curvature tensor formed by symmetric connection $P_{\underline{j k}}^{* i}$

$$
\widetilde{K}_{j m n}^{i}=P_{\underline{j m, n}}^{* i}-P_{\underline{j n, m}}^{* i}+P_{\underline{j m}}^{* p} P_{\underline{p n}}^{* i}-P_{\underline{j n}}^{* p} P_{\underline{p m}}^{* i}+P_{\underline{j m, s}}^{* i} \xi_{, n}^{* s}-P_{\underline{j n}, \dot{s}, \xi_{, m}^{* s}} .
$$

In the work [7] we find five linearly independent curvature tensors $\underset{K}{\widetilde{K}}, \ldots, \underset{5}{\widetilde{K}}$, where $\underset{1}{\widetilde{K}}, \ldots, \underset{4}{\widetilde{K}}$ given by equations $(1.7-1.10),(1.11-1.14)$, and

$$
\begin{aligned}
& \widetilde{K}_{5 m n}^{i}=\widetilde{K}_{j m n}^{i}+T_{j m}^{* p} T_{p n}^{* i}+T_{j n}^{* p} T_{p m}^{* i}, \\
& \widetilde{K}_{5}^{i}{ }_{j m n}=\frac{1}{2}\left(P_{\underline{j m, n}}^{* i}-P_{\underline{j n, m}}^{* i}+P_{j m}^{* p} P_{p n}^{* i}+P_{m j}^{* p} P_{n p}^{* i}-P_{j n}^{* p} P_{m p}^{* i}\right. \\
& \left.-P_{n j}^{* p} P_{p m}^{* i}+P_{\underline{j m, \dot{s}}}^{* p} \xi_{, n}^{s}-P_{\underline{j n, s}, \dot{s}, \xi_{, m}^{s}}^{*}\right),
\end{aligned}
$$

where $j m$ denotes symmetrization by indices $j$ and $m$.

Applying two kinds of covariant differentiation (1.5), we get

$$
\begin{aligned}
a_{j \mid m}^{i}(x, \xi) & =a_{j, m}^{i}+a_{j, \dot{p}}^{i} \xi_{, m}^{p}+P_{m p}^{* i} a_{j}^{p}-P_{j m}^{* p} a_{p}^{i} \\
& =a_{j, m}^{i}+a_{j, \dot{p}}^{i} \xi_{, m}^{p}+\left(P_{\underline{p m}}^{* i} \pm T_{p m}^{* i}\right) a_{j}^{p}-\left(P_{\underline{j m}}^{* p} \pm T_{j m}^{* p}\right) a_{p}^{i} \\
& =a_{j ; m}^{i}+T_{p m p}^{* i} a_{j}^{p}-T_{j m}^{* p} a_{p}^{i} .
\end{aligned}
$$

\section{RICCI COEFFICIENTS OF ROTATION IN A GENERALIZED FINSLER SPACE}

On Ricci coefficients of rotation in $R_{n}$ the reader can find e.g. in [20], §54. On that matter in $G R_{n}$ is written in [8]. On Ricci coefficients of rotation in $F_{n}$ see [13].

\subsection{Congruence of curves and orthogonal ennuple}

Definition 1. A congruence of curves in a $G F_{n}$ is such a family of curves that though each point of $G F_{n}$ passes one curve of the family. N mutually orthogonal congruences of curves constitute an orthogonal ennuple. Instead of congruences of curves we shall sometimes speak about congruences of the corresponding tangent vectors. 
If $\lambda_{(h)},(h=1, \ldots, N)$ are unit tangent vectors of congruences of curves of an orthogonal ennuple, then in virtue of the previous definition

$$
g_{\underline{i j}}(x, \dot{x}) \lambda_{(h)}^{i} \lambda_{(k)}^{j}=e_{(k)} \delta_{h k}, e_{(k)}= \pm 1,
$$

or

$$
e_{(k)} \lambda_{(h)}^{i} \lambda_{(k) i}=\delta_{h k},
$$

where $\delta_{h k}$ are Kronecker symbols. (of course, we do not suppose summation w.r.t. (k) in (2.1), (2.2) and in similar formulas later on.) The next theorem expresses the basic properties of orthogonal ennuples.

Theorem 1. For the unit tangent vectors $\lambda_{(h)},(h=1, \ldots, N)$ of congruences of curves of an orthogonal ennuple the relations

$$
\begin{aligned}
& \text { a) } \sum_{k=1}^{N} e_{(k)} \lambda_{(k) i} \lambda_{(k)}^{j}=\delta_{i}^{j}, \quad \text { b) } \sum_{k=1}^{N} e_{(k)} \lambda_{(k) i} \lambda_{(k) j}=g_{\underline{i j}} \\
& \text { c) } \sum_{k=1}^{N} e_{(k)} \lambda_{(k)}^{i} \lambda_{(k)}^{j}=g^{i j}
\end{aligned}
$$

are valid.

Proof. In the determinant $\operatorname{det}\left(\lambda_{(k)}^{i}\right)$, whose value is 1 , we can regard $e_{(k)} \lambda_{(k) i}$ as cofactor of the element $\lambda_{(k)}^{i}$. Developing the determinant either by rows or by columns $(2.3 a)$ follows.

Further, we have

$$
\sum_{k} e_{(k)} \lambda_{(k) i} \lambda_{(k) j}=g_{\underline{j l}} \sum_{k} e_{(k)} \lambda_{(k) i} \lambda_{(k)}^{l}=g_{\underline{j l}} \delta_{i}^{l} \Rightarrow(2.3 b) .
$$

The equation $(2.3 c)$ can be obtained in the same manner.

\subsection{Definition and basic properties of the coefficient of rotation}

Using the two kinds of covariant derivative (1.5) of a vector in a $G F_{n}$, we can define two kinds of coefficients of rotation, as two systems of invariants (for $\theta=1,2$ ).

Definition 2. The invariants

$$
\underset{\theta}{Y_{(h k m)}}(x, \dot{x}) \stackrel{\text { def }}{=} \lambda_{(h) i \mid j} \lambda_{\theta}^{i}{ }_{(k)}^{i} \lambda_{(m)}^{j}=\lambda_{(h) \mid j}^{i} \lambda_{(k) i} \lambda_{(m)}^{j}, \quad \theta=1,2 .
$$

are said to be coefficients of rotation of the given orthogonal ennuple.

From $(1.5,1.6)$ it is evidently that

$$
\lambda_{(h) \mid j}^{i}=\lambda_{(h) \mid j}^{i}, \quad \lambda_{(h) i \mid j}=\lambda_{(h) i \mid j}, \quad \lambda_{(h) \mid j}^{i}=\lambda_{(h) \mid j}^{i}, \quad \lambda_{(h) i \mid j}^{i}=\lambda_{(h) i \mid j},
$$

and it is easy to prove that there exist two kinds of coefficients of rotation in the non-symmetric case. 
Theorem 2. Both kinds of coefficients of rotation are antisymmetric in their first two indices, i.e.

$$
Y_{\theta}(h k m)=-Y_{\theta}(k h m) \Rightarrow Y_{\theta}(h k m)=0 .
$$

Proof. By covariant differentation we get from (2.2) the relation

$$
e_{(k)}\left(\lambda_{(h) \mid j}^{i} \lambda_{(k) i}+\lambda_{(h)}^{i} \lambda_{(k) i \mid j}\right)=0,
$$

from where, transvecting by $\lambda_{(m)}^{j}$,

$$
e_{(k)}\left(\lambda_{(h) \mid j}^{i} \lambda_{(k) i} \lambda_{(m)}^{j}+\lambda_{(h)}^{i} \lambda_{(k) i \mid j} \lambda_{(m)}^{j}\right) \underset{(2.4)}{=} e_{(k)}\left(Y_{\theta}(h k m)+\underset{\theta}{Y_{(k h m)}}\right)=0
$$

that is

$$
Y_{\theta}(h k m)+Y_{\theta}(k h m)=0 \Rightarrow(2.5) .
$$

Theorem 3. If

$$
Y_{(h k m)}=\lambda_{(h) i ; j} \lambda_{(k)}^{i} \lambda_{(m)}^{j}
$$

are coefficients of rotation in the associated Finsler space $F_{n}$ (see [13]), then

$$
\begin{gathered}
Y_{\theta}(h k m)=Y_{(h k m)}+(-1)^{\theta} T_{i j}^{* p} \lambda_{(h) p} \lambda_{(k)}^{i} \lambda_{(m)}^{j}, \quad \theta=1,2, \\
Y_{(h k m)}=\left(Y_{1}(h k m)+Y_{(h k m)}\right) / 2, \\
Y_{\theta}(h k h)=Y_{(h k h)}, \quad Y_{\theta}(h h k)=Y_{(h h k)}, \quad{ }_{\theta}(h k k)=Y_{(h k k)}, \quad \theta=1,2 .
\end{gathered}
$$

Proof. In virtue of (2.4) and

$$
\lambda_{i \mid n}=\lambda_{i ; n}+(-1)^{\theta} T_{i n}^{* p} \lambda_{p}, \quad \theta=1,2
$$

we have

$$
{ }_{\theta}^{Y_{(h k m)}}=\left(\lambda_{(h) i ; j}+(-1)^{\theta} T_{i j}^{* p} \lambda_{(h) p}\right) \lambda_{(k)}^{i} \lambda_{(m)}^{j} \underset{(2.6)}{\Rightarrow}(2.7) \quad \theta=1,2 .
$$

In virtue of (2.7) for two indices there follows (2.8) because for example (for $h=m$ ) is

$$
\begin{aligned}
T_{i j}^{* p} \lambda_{(h) p} \lambda_{(k)}^{i} \lambda_{(m)}^{j}=T_{p . i j}^{*} \lambda_{(h)}^{p} \lambda_{(k)}^{i} \lambda_{(h)}^{j} & =T_{j . i p}^{*} \lambda_{(h)}^{j} \lambda_{(k)}^{i} \lambda_{(h)}^{p} \\
& =-T_{p . i j}^{*} \lambda_{(h)}^{p} \lambda_{(k)}^{i} \lambda_{(h)}^{j}=0,
\end{aligned}
$$

where $T_{p . i j}^{*}=T_{i j}^{* s} g_{s \underline{p}}$. Here we applied the fact that $T_{p . i j}^{*}$ is antisymmetric in all pairs of indices. 
2.3. Expression of the derivative of the vectors of a congruence by the coefficients of rotation

Theorem 4. In $G F_{n}$ the relation

$$
\begin{aligned}
& \text { a) } \lambda_{(h) i \mid j}=\sum_{k, m=1}^{N} e_{(k)} e_{(m)} \underset{\theta}{Y}(h k m) \lambda_{(k) i} \lambda_{(m) j}, \\
& \text { b) } \lambda_{(h) \mid j}^{i}=\sum_{k, m=1}^{N} e_{(k)} e_{(m)}{\underset{\theta}{(h k m)}}_{(h)} \lambda_{(k)}^{i} \lambda_{(m) j}
\end{aligned}
$$

are valid.

Proof. a) Multiplying the relation (2.4) by $e_{(k)} e_{(m)} \lambda_{(k) p} \lambda_{(m) q}$ and summing with respect to $m, k$ get

$$
\begin{aligned}
& \sum_{k, m} e_{(k)} e_{(m)}{ }_{\theta}^{Y}(h k m) \lambda_{(k) p} \lambda_{(m) q}=\sum_{k, m} \lambda_{(h) i \mid j} \lambda_{\theta}^{i}{ }_{(k)}^{i} \lambda_{(m)}^{j} e_{(k)} e_{(m)} \lambda_{(k) p} \lambda_{(m) q} \\
& =\lambda_{(h) i \mid j}\left\{\sum_{k} e_{(k)} \lambda_{(k) p} \lambda_{(k)}^{i}\right\} \cdot\left\{\sum_{m} e_{(m)} \lambda_{(m) q} \lambda_{(m)}^{j}\right\} \\
& \underset{(2.3 a)}{=} \lambda_{(h) i \mid j} \delta_{p}^{i} \delta_{q}^{j}=\lambda_{(h) p \mid q} .
\end{aligned}
$$

Theorem 5. The covariant derivatives of vectors $\lambda_{(h) i}$ and $\lambda_{(h)}^{i}$ in the direction of the vector $\lambda_{(p)}^{j}$ can be expressed by the coefficients of rotation as linear combination of the vectors of the ennuple as follows:

$$
\text { a) } \left.\lambda_{(h) i \mid j} \lambda_{(p)}^{j}=\sum_{k} e_{(k)}{ }_{\theta}^{Y}(h k p) \lambda_{(k) i} \quad b\right) \lambda_{(h) \mid j}^{i} \lambda_{\theta}^{j}{ }_{(p)}=\sum_{k} e_{(k)}{ }_{\theta}{ }_{(h k p)} \lambda_{(k)}^{i}
$$

Proof. Transvecting the equation (2.9) by $\lambda_{(p)}^{j}$, we obtain

a) $\lambda_{(h) i j \mid j} \lambda_{(p)}^{j}=\sum_{k, m} e_{(k)} e_{(m)}{ }_{\theta}(h k p) \lambda_{(k) i} \lambda_{(m) j} \lambda_{(p)}^{j}$

$$
=\sum_{k, m} e_{(k)}{ }_{\theta}(h k p) \lambda_{(k) i} \delta_{m p}=\sum_{k} e_{(k)}{ }_{\theta}(h k p) \lambda_{(k) i} \Rightarrow(2.10) .
$$

\subsection{Integrability conditions of the equation (2.9)}

The relation (2.9) is a partial differential equation with respect to the unknown functions $\lambda_{(h) i}$. Now we are going to examine its integrability condition. 
In [10] we have obtained 10 Ricci-type identities in $G F_{n}$. In three of these identities appear the curvature tensors $\widetilde{K}, \underset{2}{K}, \widetilde{K}$, and in the others appear the quantities $\widetilde{A}, \ldots,{ }_{15}^{\widetilde{A}}$ which have the form and the role of the curvature tensors, but they are not tensors. In [5] we obtained combined Ricci-type identities, in which appear "derived" curvature tensors $\widetilde{K}_{1}^{*}, \ldots, \underset{8}{\widetilde{K}}$. In [7] is proved that only five are independent among the mentioned curvature tensors, for example $\underset{1}{\widetilde{K}}, \underset{2}{\widetilde{K}}, \underset{3}{\widetilde{K}}, \underset{4}{\widetilde{K}}, \underset{5}{\widetilde{K}}$ while the others are linear combinations of these five tensors and $\widetilde{K}$. We shall use further those of the Ricci-type identities in which appear the above tensors (the tensor $\widetilde{K}_{1}^{*}$ is linear combination of $\underset{1}{\widetilde{K}}, \underset{2}{\widetilde{K}}$, while the tensor $\widetilde{K}_{2}^{*}$ does not appear in identities which we need.)

Theorem 6. In $G F_{n}$ the first two integrability conditions $(\theta=1,2)$ of equations (2.9) are

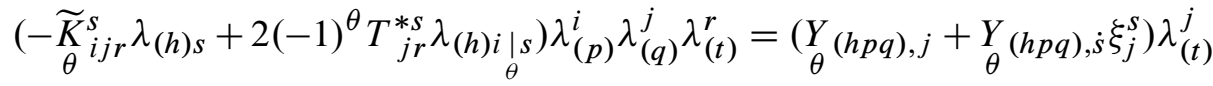

$$
\begin{aligned}
& -\left(Y_{\theta}(h p t), j+Y_{\theta}(h p t), \dot{s} \xi_{j}^{S}\right) \lambda_{(q)}^{j}+\sum_{k=1}^{N} e_{(k)}\left\{Y_{\theta}(h k q){ }_{\theta}^{Y}(k p t)-Y_{\theta}(h k t){ }_{\theta}(k p q)\right. \\
& \left.+Y_{\theta}(h p k)\left[Y_{\theta}(k q t)-Y_{\theta}(k t q)\right]\right\}, \quad \theta=1,2
\end{aligned}
$$

Proof. Applying the Ricci-type identities from [10], we have

$$
\lambda_{(h) i \mid j r}-\lambda_{(h) i \mid r j}=-\widetilde{K}_{\theta}^{s}{ }_{i j r} \lambda_{(h) s}+2(-1)^{\theta} T_{j r}^{* s} \lambda_{(h) i \mid s}, \quad \theta=1,2,
$$

By repeated differentiation of (2.9) one can form the difference on the left side of this equation, and then (2.11) easily follows.

Theorem 7. The third integrability condition of the equation (2.9) in $G F_{n}$ is

$$
\begin{aligned}
& -\widetilde{K}_{3}^{s}{ }_{i j r} \lambda_{(h) s} \lambda_{(p)}^{i} \lambda_{(q)}^{j} \lambda_{(t)}^{r}=\left(Y_{1}(h p q), j+Y_{1}(h p q) \dot{s} \xi_{j}^{s}\right) \lambda_{(t)}^{j} \\
& -\left(Y_{2}(h p t), j+Y_{2}(h p t) \dot{s} \xi_{j}^{S}\right) \lambda_{(q)}^{j}+\sum_{k=1}^{N} e_{(k)}\left[Y_{1}(h k q) \underset{2}{Y}(k p t)-Y_{2}(h k t) \underset{1}{Y}(k p q)\right. \\
& \left.+Y_{1}(h p k) \underset{2}{Y}(k q t)-Y_{2}(h p k) \underset{1}{Y}(k t q)\right] \text {. }
\end{aligned}
$$

where $\underset{3}{\widetilde{K}}$ is given by (1.9). 
Proof. Applying the corresponding identity from [10] we get

$$
\lambda_{(h) i|j| r} \operatorname{li}_{2}-\lambda_{(h) i|r| j}=-\widetilde{K}_{3}^{s}{ }_{i j r} \lambda_{(h) s} .
$$

Further, we use (2.9) to form the difference on the left side.

Theorem 8. The fourth integrability condition of the equation (2.9) is

$$
\begin{aligned}
& \widetilde{K}_{4}^{i}{ }_{s j r} \lambda_{(h)}^{s} \lambda_{(p) i} \lambda_{(q)}^{j} \lambda_{(t)}^{r}=\left(Y_{1}(h p q), j+Y_{1}(h p q) \dot{s} \xi_{j}^{S}\right) \lambda_{(t)}^{j} \\
& -\left(Y_{2}(h p t), j+Y_{2}(h p t) \dot{s} \xi_{j}^{S}\right) \lambda_{(q)}^{j}+\sum_{k=1}^{N} e_{(k)}\left[Y_{1}(h k q) \underset{2}{Y}(k p t)+Y_{1}(h p k){ }_{1}^{Y}(k q t)\right. \\
& \left.-Y_{2}(h k t){ }_{1}(k p q)-Y_{2}(h p k) \underset{2}{Y}(k t q)\right] \text {. }
\end{aligned}
$$

where $\widetilde{K}_{4}$ is given by (1.10).

Proof. Using by equations (1.10) we see that

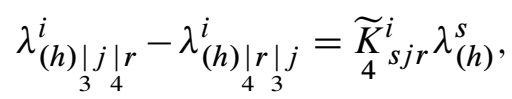

and the use of (2.9) yields the integrability condition (2.13).

Theorem 9. The fifth integrability condition of the equation (2.9) is

$$
\begin{aligned}
& \widetilde{K}_{2}^{* i}{ }_{s j r} \lambda_{(h)}^{s} \lambda{ }_{(p) i} \lambda_{(q)}^{j} \lambda_{(t)}^{r}=\left(Y_{1}\left(h p[q), j+Y_{1}{ }_{(h p[q) \dot{s}} \xi_{j}^{S}\right) \lambda_{(t)]}^{j}\right. \\
& -\left(Y _ { 2 } \left(h p[q), j+Y_{2}\left(h p[q) \dot{s} \xi_{j}^{S}\right) \lambda_{(t)]}^{j}+\sum_{k} e_{(k)}\left[Y_{1}(h k q){ }_{1}^{Y}(k p t)+Y_{1}(h p k) Y_{1}(k q t)\right.\right.\right. \\
& -Y_{1}(h k t){ }_{2}(k p q)-Y_{1}(h k t) \underset{2}{Y}(k p q)+Y_{2}(h k q) \underset{2}{Y}(k p t)+Y_{2}(h p k) \underset{2}{Y}(k q t) \\
& \left.-Y_{2}(h k t){ }_{1}^{Y}(k p q)-Y_{2}(h k t){ }_{1}^{Y}(k p q)\right] \text {, }
\end{aligned}
$$

where $[q t]$ we denoted symmetrization without division by indices $q$ and $t$ and $\widetilde{K}_{2}^{* i} \widetilde{s i r}^{* i}$ $\widetilde{K}_{5}^{i}$ sjr given by (1.16).

Proof. We use that Ricci-type identity in which the curvature $\widetilde{K}_{2}^{*}$ appears. From corresponding identities in [5] we have

$$
\lambda_{(h) \mid j r}^{i}-\lambda_{(h) \mid \underset{1}{|r| j}}^{i}+\lambda_{(h)|j|}^{i}-\lambda_{(h)|r| j}^{i}=2 \underset{2}{i} \widetilde{K}_{s j r}^{* i} \lambda_{(h)}^{s},
$$

and then use (2.9). 


\section{GEODESIC MAPPINGS}

Definition 3. Geodesic in $G F_{n}$ is given by

$$
\frac{d^{2} x^{i}}{d s^{2}}+P_{j k}^{* i}\left(x, \frac{d x}{d s}\right) \frac{d x^{j}}{d s} \frac{d x^{k}}{d s}=0 .
$$

Consider two $N$-dimensional spaces of non-symmetrical affine connection: $G F_{n}$ and $G \bar{F}_{n}$. So, if connection coefficients of these spaces are respectively $P_{j k}^{* i}$ and $\bar{P}_{j k}^{* i}$, we suppose that in general the symmetry with respect to indices $j, k$ is not in force.

One says that reciprocal one valued mapping $f: G F_{n} \rightarrow G \bar{F}_{n}$ is geodesic, if geodesics of the space $G F_{n}$ pass to geodesics of the space $G \bar{F}_{n}$. We can consider these spaces in the common by this mapping system of local coordinates, i.e. for $f: M \mapsto \bar{M}$ we have $M\left(x^{1}, \ldots, x^{n}, \dot{x}^{1}, \ldots, \dot{x}^{n}\right) \equiv M(x, \dot{x})$ and $\bar{M}\left(x^{1}, \ldots, x^{n}, x^{1}, \ldots\right.$, $\left.x^{n}, \dot{x}^{1}, \ldots, \dot{x}^{n}\right) \equiv \bar{M}(x, \dot{x})$, where $M \in G F_{n}, \bar{M} \in G \bar{F}_{n}$. In the corresponding points $M(x, \dot{x})$ and $\bar{M}(x, \dot{x})$ we can put

$$
\bar{P}_{j k}^{* i}=P_{j k}^{* i}+D_{j k}^{i}, \quad(i, j, k=1, \ldots, N),
$$

where $D_{j k}^{i}(x)$ is the deformation tensor of the connection $P^{*}$ of $G F_{n}$ according to the mapping $f: G F_{n} \rightarrow G \bar{F}_{n}$.

Definition 4. The curve

$$
l: x^{i}=x^{i}(t)
$$

is geodesic of $G F_{n}$ if and only if is:

$$
\ddot{x}^{i}+P_{p q}^{* i} \dot{x}^{p} \dot{x}^{q}=\rho(t) \dot{x}^{i}(t),
$$

where $\rho(t)$ is an invariant and $t$ an arbitrary parameter.

If $f: l \rightarrow \bar{l}$, then in the common by the mapping $f$ coordinates $\bar{x}^{i} \equiv x^{i}$ it is $\dot{\bar{x}}^{i}=d \bar{x}^{i} / d t=\dot{x}^{i}$, and $\bar{l}$ is geodesic in $G \bar{F}_{n}$ too, from where we get

$$
\ddot{x}^{i}+\bar{P}_{p q}^{* i} \dot{x}^{p} \dot{x}^{q}=\bar{\rho}(t) \dot{x}^{i}(t)
$$

Subtracting (3.3) and (3.4), we obtain

$$
\left(\bar{P}_{p q}^{* i}-P_{p q}^{* i}\right) \dot{x}^{p} \dot{x}^{q}=(\bar{\rho}(t)-\rho(t)) \dot{x}^{i}(t),
$$

and, because of (3.2):

$$
D_{p q}^{i} \dot{x}^{p} \dot{x}^{q}=2 \psi(t) \dot{x}^{i}(t)
$$

where $\psi(t)=(\bar{\rho}(t)-\rho(t)) / 2$. Denoting by $D_{\underline{j k}}^{i}, D_{j k}^{i}$ the symmetric and antisymmetric part of $D_{j k}^{i}$ respectively, we get

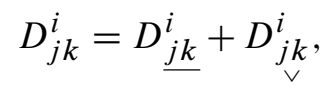


and (3.5) reduces to

$$
D_{\underline{p q}}^{i} \dot{x}^{p} \dot{x}^{q}=2 \psi(t) \dot{x}^{i}(t)
$$

As in the case of a symmetric connection (see for ex. [4]) one concludes that

$$
\psi(t)=\psi_{p}\left(x^{1}(t), \ldots, x^{n}(t), \dot{x}^{1}(t), \ldots, \dot{x}^{n}(t)\right) \dot{x}^{p}(t),
$$

and from (3.6):

$$
D_{\underline{p q}}^{i} \dot{x}^{p} \dot{x}^{q}=2 \psi_{p} \dot{x}^{p} \dot{x}^{i}=\psi_{p} \dot{x}^{p} \dot{x}^{q} \delta_{q}^{i}+\psi_{q} \dot{x}^{p} \dot{x}^{q} \delta_{p}^{i}=\left(\psi_{p} \delta_{q}^{i}+\psi_{q} \delta_{p}^{i}\right) \dot{x}^{p} \dot{x}^{q},
$$

wherefrom

$$
D_{\underline{j k}}^{i}=\delta_{j}^{i} \psi_{k}+\delta_{k}^{i} \psi_{j}
$$

Denoting also

$$
D_{j k}^{i}=\xi_{j k}^{i}=-\xi_{k j}^{i},
$$

by substitution in (3.2) we obtain

$$
\bar{P}_{j k}^{* i}=P_{j k}^{* i}+\delta_{j}^{i} \psi_{k}+\delta_{k}^{i} \psi_{j}+\xi_{j k}^{i},
$$

and the deformation tensor is

$$
D_{j k}^{i}=\delta_{j}^{i} \psi_{k}+\delta_{k}^{i} \psi_{j}+\xi_{j k}^{i} .
$$

So, the condition (3.8) is necessary that the mapping $f$ be geodesic. It is easily to prove that this condition is sufficient too, and we have

Theorem 10. A necessary and sufficient condition that the mapping $f: G F_{n} \rightarrow$ $G \bar{F}_{n}$ be geodesic is the deformation tensor $D_{j k}^{i}$ from (3.2) at the mapping $f$ to has the form (3.9), where $\psi_{j}(x, \dot{x})$ is a covariant vector, and $\xi_{j k}^{i}(x, \dot{x})$ an antisymmetric tensor.

For $k=i$, we obtain from (3.7):

$$
D_{\underline{j i}}^{i}=\delta_{j}^{i} \psi_{i}+\delta_{i}^{i} \psi_{j}=\psi_{j}+N \psi_{j},
$$

wherefrom

$$
\psi_{i}=\frac{1}{N+1} D_{i \underline{p}}^{p},
$$

which, by substitution in (3.8), gives

$$
\bar{P}_{j k}^{* i}=P_{j k}^{* i}+\frac{1}{N+1}\left(\delta_{j}^{i} D_{\underline{k p}}^{p}(x, \dot{x})+\delta_{k}^{i} D_{\underline{j p}}^{p}(x, \dot{x})\right)+D_{\substack{v \\ i}}^{i}(x, \dot{x}),
$$

where $D_{j k}^{i}(x)$ is the deformation tensor.

On the base of above explained, we get 
Theorem 11. Let a space $G F_{n}$ be given, i.e. on a differentiable manifold $M_{n}$ let be defined non-symmetric connection coefficients $P_{j k}^{* i}(x, \dot{x})$. If on $M_{n}$ is given a tensor $D_{j k}^{i}(x, \dot{x})$ too and we determine $\bar{P}_{j k}^{* i}(x, \dot{x})$ according to (3.11), then on $M_{n}$ will be defined a space $G \bar{F}_{n}$, with connection coefficients $\bar{P}_{j k}^{* i}$, and then $G F_{n}$ and $G \bar{F}_{n}$ have common geodesics. We obtain the same result (on the base of (3.8) by choice of a vector $\psi_{i}(x, \dot{x})$ and antisymmetric tensor $\xi_{j k}^{i}(x, \dot{x})=D_{j k}^{i}(x, \dot{x})$.

The question forces itself: Is it possible a geodesic mapping of a space $G F_{n}$ with a non-symmetric affine connection on to a space $\bar{F}_{n}$ with a symmetric affine connection? It is easy to see that the next theorem is valid:

Theorem 12. A necessary and sufficient condition that a mapping $f: G F_{n} \rightarrow \bar{F}_{n}$ of a non-symmetric affine connection space $G F_{n}$ onto a symmetric affine connection space $\bar{F}_{n}$ be geodesic, is

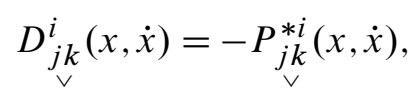

where $D_{j k}^{i}(x, \dot{x}), P_{j k}^{* i}(x, \dot{x})$, are antisymmetric parts of the deformation tensor and connection coefficients of the $G F_{n}$ respectively.

Remark 1. It is easy to check that a set of geodesic mappings of a space $G F_{n}$ makes a group.

\section{SOME PROJECTIVE INVARIANTS OF GEODESIC MAPPINGS}

Putting $D$ into (3.11) in accordance with (3.2) we get

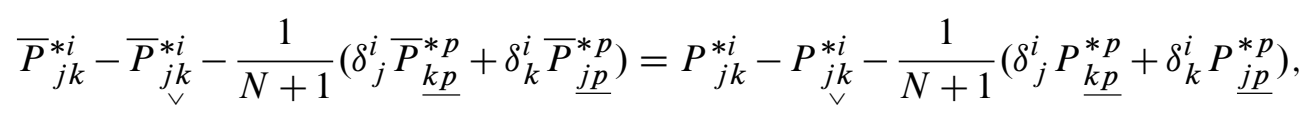

Denoting

$$
\mathcal{T}_{j k}^{i}=P_{\underline{j k}}^{* i}-\frac{1}{N+1}\left(\delta_{j}^{i} P_{\underline{k p}}^{* p}+\delta_{k}^{i} P_{\underline{j p}}^{* p}\right)=\mathcal{T}_{k j}^{i},
$$

we see that

$$
\overline{\mathcal{T}}_{j k}^{i}=\mathcal{T}_{j k}^{i} .
$$

The magnitudes $\mathcal{T}_{j k}^{i}$ we call generalized Thomas's projective parameters at the mapping $f: G F_{n} \rightarrow G \bar{F}_{n}$. Accordingly, these magnitudes are invariant at a geodesic mapping. Starting from $(4.1,4.2)$, one obtains $(3.11)$, and we conclude that the next theorem is valid:

Theorem 13. A necessary and sufficient condition that a mapping $f: G F_{n} \rightarrow$ $G \bar{F}_{n}$ be geodesic is that generalized Thomas's projective parameters (4.1) are invariant, that is (4.2) to be valid. 
Theorem 14. If $\lambda_{(h)}^{i}(x)$ and $\lambda_{(h) i}(x)$ are the contravariant, respectively covariant components of an orthogonal ennuple, then the following geometric entities are invariant under the geodesic mapping:

$$
\underset{\theta}{\mathcal{M}_{k}^{i}}(x, \dot{x})=\lambda_{(h) \mid}^{i}(x)-\frac{1}{n+1} \lambda_{(h)}^{p}(x) \sum_{h} e_{(h)} \lambda_{(h) q} \delta_{(p}^{q} \lambda_{(h) \mid k)}, \theta=1,2,
$$

and

$$
\underset{\theta}{\mathcal{M}^{*}}{ }_{k}(x, \dot{x})=\sum_{h} e_{(h)} \lambda_{(h) p} \lambda_{(h) \mid k}^{p}-D_{\underline{k q}}^{q}, \theta=1,2,
$$

where $(p \ldots k)$ denotes symmetrization by respect of indices $p$ and $k$.

Proof. If we denote by $\lambda_{(h) \mid \bar{\theta}}^{i}, \theta=1,2$ the covariant derivative wrt the connection $\bar{P}^{*}$ in sense of (1), we have

$$
\lambda_{\substack{(h) \mid \bar{k} \\ 1 \\ 2}}^{i}=\lambda_{(h), k}^{i}+\lambda_{(h)}^{p} \bar{P}_{\substack{p k \\ k p}}^{* i}
$$

Using $(3.8,4.1)$ we have

$$
\begin{aligned}
& \lambda_{(h) \mid \bar{k}}^{i}-\lambda_{(h) \mid k}^{i}=\lambda_{(h)}^{p}\left(\delta_{p}^{i} \psi_{k}+\delta_{k}^{i} \psi_{p}+\xi_{p k}^{i}\right) \\
& \lambda_{(h) \mid \bar{k}}^{i}-\lambda_{(a) \mid k}^{i}=\lambda_{(h)}^{p}\left(\delta_{k}^{i} \psi_{p}+\delta_{p}^{i} \psi_{k}+\xi_{k p}^{i}\right) .
\end{aligned}
$$

Multiplying (4.6) by $\lambda_{(h) i}$ and summing with respect to indices $h$ and using orthogonality condition, we have

$$
\begin{aligned}
& \sum_{h} e_{(h)} \lambda_{(h) p}\left(\lambda_{(h) \mid \bar{k}}^{p}-\lambda_{(h) \mid k}^{p}\right)=(N+1) \psi_{k} \\
& \sum_{h} e_{(h)} \lambda_{(h) p}\left(\lambda_{(h) \mid \bar{k}}^{p}-\lambda_{(h) \mid k}^{p}\right)=(N+1) \psi_{k} .
\end{aligned}
$$

Eliminating the $\psi_{k}$ from (4.6) and (4.7) we get

$$
\lambda_{\substack {(h) \mid \bar{k} \\
\begin{subarray}{c}{1 \\
2{ ( h ) | \overline { k } \\
\begin{subarray} { c } { 1 \\
2 } }\end{subarray}}^{i} \lambda_{\substack{(h) \mid k \\
1}}^{i}=\frac{1}{n+1} \lambda_{(h)}^{p}\left(\sum_{h} e_{(h)} \lambda_{(h) q} \delta_{(p}^{q} \lambda_{(h) \mid \bar{k})}-\sum_{h} e_{(h)} \lambda_{(h) q} \delta_{(p}^{q} \lambda_{(h) \mid k)}\right) \text {. }
$$

With help (3.10) we get (4.3).

Theorem 15. When $G F_{n}$ and $G \bar{F}_{n}$ are in geodesic correspondence, we have the following projective invariant entities:

$$
S_{\theta} h k m(x, \dot{x})=Y_{\theta}(h k m)-\frac{1}{n+1}\left(\delta_{h k} \lambda_{(m)}^{k} P_{k i}^{* i}+\delta_{k m} \lambda_{(h)}^{j} P_{j i}^{* i}\right), \theta=1,2,
$$


where $Y_{\theta}(\mathrm{hkm}) \theta=1,2$, are Ricci coefficients of rotation.

Proof. Using (4.5) we get

$$
\lambda_{\substack {(h) \mid \bar{k} \\
\begin{subarray}{c}{1 \\
2{ ( h ) | \overline { k } \\
\begin{subarray} { c } { 1 \\
2 } }\end{subarray}}^{i}-\lambda_{\substack {(h) \mid k \\
\begin{subarray}{c}{1 \\
2{ ( h ) | k \\
\begin{subarray} { c } { 1 \\
2 } }\end{subarray}}^{i}=\frac{1}{N+1} \lambda_{(h)}^{j}\left(\delta_{j}^{i} D_{\underline{k p}}^{p}(x, \dot{x})+\delta_{k}^{i} D_{\underline{j p}}^{p}(x, \dot{x})\right) .
$$

Multiplying (4.8) by $\lambda_{(k) i} \lambda_{(m)}^{k}$ and using orthogonality condition, we have

$$
\begin{aligned}
& \bar{Y}_{2} h k m-\frac{1}{n+1}\left(\delta_{h k} \lambda_{(m)}^{k} \bar{P}_{k i}^{* i}+\delta_{k m} \lambda_{(h)}^{q} \bar{P}_{q p}^{* p}\right) \\
& =\underset{1}{Y_{h k m}}-\frac{1}{n+1}\left(\delta_{h k} \lambda_{(m)}^{k} P_{k p}^{* p}+\delta_{k m} \lambda_{(h)}^{j} P_{j p}^{* p}\right),
\end{aligned}
$$

where the projectively transformed Ricci coefficients of rotation are given by

$$
\bar{Y}_{\theta} h k m=\lambda_{(h) p \mid \bar{\theta}} \lambda_{(k)}^{p} \lambda_{(m)}^{q}=\lambda_{(h) \mid \bar{q}^{\prime}}^{p} \lambda_{(k) p} \lambda_{(m)}^{q}, \quad \theta=1,2 .
$$

\section{ACKNOWLEDGEMENT}

The authors are grateful to the Professor Vladimir Balan for valuable comments and suggestion references, which were most helpful in improving the manuscript.

\section{REFERENCES}

[1] V. Balan and N. I. Rodica, "Berwald-moor metrics and structural stability of conformally deformed geodesic SODE," Appl. Sci., vol. 11, pp. 19-34, 2009.

[2] L. P. Eisenhart, "Generalized Riemannian spaces," Proc. Nat. Acad. Sci. USA, vol. 37, pp. 311315, 1951, doi: 10.1073/pnas.37.5.311.

[3] H. Goenner, On the History of Unified Field Theories. Part, ii ed. Living Reviews in Relativity: Living Rev. Relativity, 2014, vol. 7.

[4] J. Mikeš, S. Báscó, and V. Berezovski, "Geodesic mappings of weakly Berwald spaces and Berwald spaces onto Riemannian spaces," Int. J. Pure Appl. Math., vol. 45, no. 3, pp. 413-418, 2008.

[5] S. M. Minčić, "Curvature tensors of the space of non-symmetric affine connexion, obtained from the curvature pseudotensors," Mat. vesnik, vol. 13(28), no. 4, pp. 421-435, 1976.

[6] S. M. Minčić, "New commutation formulas in the non-symmetric affine connection space," Publ. Inst. Math. (Beograd) (N. S), vol. 22(36), pp. 189-199, 1977.

[7] S. M. Minčić, "Independent curvature tensors and pseudotensors of spaces with non-symmetric affine connection," Coll. Math. Soc. János Bolyai, vol. 31, pp. 445-460, 1979.

[8] S. M. Minčić, "Ricci coefficients of rotation in a generalized Riemannian space," Publ. Math. Debrecen, vol. 41, no. 3-4, pp. 173-180, 1992.

[9] S. M. Minčić and M. L. Zlatanović, "New commutation formulas for $\delta$-differentation in generalized Finsler space," Differ. Geom. Dyn. Syst., vol. 12, pp. 145-157, 2010.

[10] S. M. Minčić and M. L. Zlatanović, "Commutation formulas for $\delta$-differentation in a generalized Finsler space," Kragujevac J. Math., vol. 35, no. 2, pp. 277-289, 2011. 
[11] S. M. Minčić and M. L. Zlatanović, "Geometric interpretation of the torsion tensor, curvature tensors and pseudotensors in generalized Finsler space (in Russian)," Izv. Vyssh. Uchebn. Zaved. Mat., vol. 1, pp. 31-40, 2013.

[12] R. Miron and M. Anastasiei, Vector bundles and Lagange spaces with applications to relativity. Geometry Balkan Press, 1997.

[13] H. D. Pande, "Projective invariants of an orhogonal ennuple in a Finsler space," Annales de l'institut Fourier, vol. 18, no. 2, pp. 337-342, 1968, doi: 10.5802/aif.304.

[14] H. D. Pande and K. K. Gupta, "Bianchi's identites in a Finsler space with non-symmetric connection," Bull. Acad. Serbe Sci. Arts Cl. Sci. Math. Natur. (N.S.), vol. 1, no. 10, pp. 41-46, 1979.

[15] S. Rastogi, "On projective invariants based on non-linnear connnection in a Finsler space," Publ. Math. Debrecen, vol. 28, no. 1-2, pp. 121-128, 1981.

[16] S. Rastogi, "On projective invariants based on non-linnear connnection in a Finsler space," International Centre For Theoretical Physics, Trieste, Italy, pp. 1-8, 1986.

[17] H. Rund, The Differential Geometry of Finsler Space (in Russian). Moskow, 1981.

[18] A. C. Shamihoke, "Some properties of a curvature tensors in a generalised Finsler space," Tensor (N.S.), vol. 12, pp. 97-109, 1962.

[19] A. C. Shamihoke, "A note on a curvature tensor in a generalized Finsler space," Tensor, N.S., vol. 15 , pp. 20-22, 1964.

[20] C. E. Weatherburn, Riemannian geometry and tensor calculus. Cambridge U. P., 1950.

[21] M. L. Zlatanović and M. Cvetković, "New Cartan's tensors and pseudotensors in a generalized Finsler space," Filomat, vol. 28, no. 1, pp. 107-117, 2014.

[22] M. L. Zlatanović and S. M. Minčić, "Identities for curvature tensors in generalized Finsler space," Filomat, vol. 23, no. 2, pp. 34-42, 2009, doi: 10.2298/FIL0902034Z.

Authors' addresses

Svetislav M. Minčić

University of Niš, Faculty of Science and Mathematics, Višegradska 33, 18000 Niš, Serbia

E-mail address: svetislavmincic@yahoo.com

Mića S. Stanković

University of Niš, Faculty of Science and Mathematics, Višegradska 33, 18000 Niš, Serbia

E-mail address: stmica@ptt.rs

Milan Lj. Zlatanović

University of Niš, Faculty of Science and Mathematics, Višegradska 33, 18000 Niš, Serbia

E-mail address: zlatmilan@yahoo.com 